\title{
TWO-BRIDGE LINKS WITH UNLINKING NUMBER ONE
}

\author{
PETER KOHN
}

(Communicated by Frederick R. Cohen)

\begin{abstract}
Three conditions equivalent to a two-bridge link having unlinking number one are given. As a corollary it is shown that an unknotting crossing appears in the minimal diagram of a two-bridge knot or link. In addition, the absolute value of the linking number of the components is computed for all unlinking number one two-bridge links.
\end{abstract}

\section{INTRODUCTION}

Let $L$ be a one- or two-component link in $S^{3}$. The unlinking (or unknotting) number $u(L)$ of the link $L$ is the minimal number of crossing changes necessary to convert $L$ into a trivial link (or knot), where this minimum is taken over all diagrams of $L$. Now, let $L=S(p, q)$ be the two-bridge link whose two-fold branched cover is the lens space $L(p, q)$, where $p$ and $q$ are relatively prime and $p$ is positive. The number of components of $L$ corresponds to the parity of $p$ [Sieb]. When $p$ is even, $L$ has two components, for $p$ odd, $L$ is a knot. We write

$$
\frac{p}{q}=\left[a_{0}, a_{1}, \ldots, a_{r}\right],
$$

where $\left[a_{0}, a_{1}, \ldots, a_{r}\right]$ denotes the continued fraction

$$
a_{0}+\frac{1}{a_{1}+\frac{1}{a_{2}+\frac{1}{a_{3}+\cdot \cdot+\frac{1}{a_{r}}}}}
$$

Let $C\left(a_{0}, a_{1}, \ldots, a_{r}\right)=1^{*} . a_{0} a_{1} \ldots a_{r}$ as in Conway's notation [C] for a diagram of a two-bridge link. $S(p, q)$ and $S\left(p^{\prime}, q^{\prime}\right)$ are equivalent (i.e., isotopic) as links if and only if $p=p^{\prime}$ and $q^{ \pm 1} \equiv q^{\prime} \bmod p$ [S]. Furthermore, $C\left(a_{0}, a_{1}, \ldots, a_{r}\right)$ and $S(p, q)$ are equivalent if

$$
\frac{p}{q}=\left[a_{0}, a_{1}, \ldots, a_{r}\right] \text {. }
$$

Received by the editors April 9, 1990; this paper was presented at the 863rd meeting of the AMS in January 1991.

1980 Mathematics Subject Classification (1985 Revision). Primary 57M25.

(C) 1991 American Mathematical Society $0002-9939 / 91 \$ 1.00+\$ .25$ per page 
Herein we prove the following:

Theorem 1. For $L$ a nontrivial two-bridge link, the following are equivalent:

(i) $u(L)=1$;

(ii) There exist relatively prime integers $m$ and $n$ such that $L$ is equivalent to $S\left(2 n^{2}, 2 m n \pm 1\right)$;

(iii) $L$ can be expressed as $C\left(a_{0}, a_{1}, \ldots, a_{k}, \pm 2,-a_{k}, \ldots,-a_{1},-a_{0}\right)$;

(iv) $L$ can be expressed as either

$$
\begin{aligned}
& \quad C\left(c_{0}, c_{1}, \ldots, c_{r-1}, c_{r}, 1,1, c_{r}-1, c_{r-1}, \ldots, c_{1}, c_{0}\right) \text { or } \\
& \quad C\left(c_{0}, c_{1}, \ldots, c_{r-1}, c_{r}-1,1,1, c_{r}, c_{r-1}, \ldots, c_{1}, c_{0}\right), \\
& \text { where } c_{i} \geq 1 \text { for } i=0,1, \ldots, r \text { and } c_{r} \geq 2 .
\end{aligned}
$$

Kanenobu and Murakami [KM] proved the analogous theorem for two-bridge knots:

Theorem 2. For $K$ a nontrivial two-bridge knot, the following conditions are equivalent:

(i) $u(K)=1$;

(ii) There exist an odd integer $p$ and relatively prime integers $m$ and $n$ with $2 m n=p \pm 1$ and $K$ is equivalent to $S\left(p, 2 n^{2}\right)$;

(iii) $K$ can be expressed as $C\left(a_{0}, a_{1}, \ldots, a_{k}, \pm 2,-a_{k}, \ldots,-a_{1}\right)$.

A similar fourth condition may be added to Theorem 2 :

(iv) $K$ can be expressed as one of the following, or its mirror image:

$$
\begin{gathered}
C\left(c_{0}, c_{1}, \ldots, c_{r-1}, c_{r}, 1,1, c_{r}-1, c_{r-1}, \ldots, c_{1}\right) \text { or } \\
C\left(c_{0}, c_{1}, \ldots, c_{r-1}, c_{r}-1,1,1, c_{r}, c_{r-1}, \ldots, c_{1}\right),
\end{gathered}
$$

where $c_{i} \geq 1$ for $i=0,1, \ldots, r$ and $c_{r} \geq 2$.

Remark. The advantage of this condition is that this diagram for $S(p, q)$ can be calculated using the standard Euclidean algorithm. This diagram will be alternating and therefore minimal [Mu]. A knot or link with unknotting number one may be recognized from this diagram alone. In addition, the unknotting crossing appears in this diagram.

This paper is organized as follows. In $\S 2$ we establish some terminology and prove several lemmata concerning unlinking two-component links and surgery on $S^{2} \times S^{1}$. In $\S 3$ we discuss continued fractions via the Euler bracket function. The proof of Theorem 1 is found in $\S 4$.

I would like to thank Professor Cameron McA. Gordon for his helpful comments and suggestions in the preparation of this paper.

\section{Preliminaries}

Let $N(K)$ be the regular neighborhood of the knot $K$ in a closed orientable 3-manifold $M$, with $\mu$ a meridian of $N(K)$. Let $X$ be the exterior of $K$ in 
$M$, that is,

$$
X=M-\text { int } N(K) .
$$

Now, let $M(r)$ denote the closed manifold obtained by attaching a solid torus, $V$, to $X$ so that a curve of slope $r$ on $\partial X$ bounds a disk in $V$. Here the slope indicates the isotopy class of a nontrivial simple closed curve in $\partial X$. We shall say that $M(r)$ is the result of $r$-surgery on $K$ in $M$. For two slopes $r$ and $s$ in $\partial X$, let $\Delta(r, s)$ be their minimal geometric intersection number. We shall use $M_{L}$ to denote the two-fold covering of $S^{3}$ branched over the link $L$.

Lemma 3. If $L$ is a link with two components and $u(L)=1$, then $M_{L}$ is obtained by r-surgery on some knot in $S^{2} \times S^{1}$, where $\Delta(r, \mu)=2$.

Proof. This follows immediately from two facts. The proof of Lemma 1 of [L] explains the case of knots having unknotting number one. We combine this with the fact that the double branched covering of $S^{3}$ along a trivial two-component link is $S^{2} \times S^{1}$.

Throughout the proof of the following lemma we adopt the notation and terminology of [JN]. The Seifert invariants for a Seifert fibration have the form

$$
M\left(g ;\left(\alpha_{1}, \beta_{1}\right),\left(\alpha_{2}, \beta_{2}\right), \ldots,\left(\alpha_{m}, \beta_{m}\right)\right),
$$

where $g$ is the genus of the orbit surface, $F$, and $m$ is the number of surgery instructions used to obtain the Seifert fibered manifold from the genuine $S^{1}$ bundle over $F$. Our convention shall be that when $g$ is nonnegative, $F$ is orientable, while $g$ negative implies that $F$ is nonorientable $\left(F \cong \#_{i=1}^{|g|} \mathbf{R} P^{2}\right)$. Each pair $\left(\alpha_{i}, \beta_{i}\right)$ specifies a particular surgery. If each $\alpha_{i}$ is nonzero, we obtain a true Seifert fibered manifold. However, if $\alpha_{i}=0$ for some $i$, we obtain a "generalized" Seifert fibered space.

We shall be using the fact that the only true Seifert fibrations for $S^{2} \times S^{1}$ are of the form $M(0 ;(\alpha, \beta),(\alpha,-\beta))$. The fundamental group of an arbitrary Seifert manifold may be calculated from its invariants. Using such calculations, we find that for $\pi_{1}(M)$ to be $\mathrm{Z}$, the invariants must be as stated above. When $S^{2} \times S^{1}$ is fibered as above we say that $S^{2} \times S^{1}$ is fibered by $(\alpha, \beta)$-torus knots or $(\alpha, \beta)$-curves.

Lemma 4. Let $K$ be a knot in $S^{2} \times S^{1}$ and let $M=S^{2} \times S^{1}-N(K)$. If $M$ is Seifert fibered, then $K$ is an ordinary fiber in some Seifert fibration of $S^{2} \times S^{1}$. Proof. Let $V$ be a solid torus with meridian $\mu$. By hypothesis $M$ is Seifert fibered and $S^{2} \times S^{1}$ is the union of $M$ and $V$ along the boundary. There are two cases to consider. Either $\mu$ is identified with a fiber of $M$ or it is not. When $\mu$ is not identified with a fiber, the fibration of $M$ extends to $V$ [Seif]. The core of $V$ is isotopic to $K$ in $S^{2} \times S^{1}$. K is a fiber in this fibration. Even if $K$ is an exceptional fiber, we may re-fiber $S^{2} \times S^{1}$ as a trivial $S^{1}$-bundle over $S^{2}$ so that $K$ is isotopic to an ordinary fiber. 
When $\mu$ is identified with a fiber, we have a generalized Seifert fibration of $S^{2} \times S^{1}$. We claim that the orbit surface $F$ must be nonorientable. To see this, assume that $F$ is orientable. The first homology of an orientable generalized Seifert fibered manifold with an orientable orbit surface is

$$
\begin{array}{r}
G=\left\langle A_{1}, B_{1}, \ldots, A_{g}, B_{g}, Q_{1}, \ldots, Q_{m}, H\right| \alpha_{i} Q_{i}+\beta_{i} H=0, i=1, \ldots, m, \\
\left.Q_{1}+\cdots+Q_{m}=0\right\rangle \\
\cong \mathbf{Z}^{2 g} \oplus\left\langle Q_{1}, \ldots, Q_{m}, H\right| \alpha_{i} Q_{i}+\beta_{i} H=0, i=1, \ldots, m, \\
\left.Q_{1}+\cdots+Q_{m}=0\right\rangle .
\end{array}
$$

Here, since $M \cup V=S^{2} \times S^{1}$ and $G \cong H_{1}\left(S^{2} \times S^{1}\right) \cong \mathbf{Z}$ we must have that $g=0$. Furthermore, since $M$ is Seifert fibered, we may assume without loss of generality that $\alpha_{i} \neq 0$ for $i=1, \ldots, m-1$ and that $\alpha_{m}=0, \beta_{m}=1$ (that is, the $m$ th surgery on the trivial $S^{1}$-bundle sends the meridian to the fiber). Thus the relation $\alpha_{m} Q_{m}+\beta_{m} H=0$ gives us $H=0$. The group

$$
G \cong\left\langle Q_{1}, \ldots, Q_{m} \mid \alpha_{i} Q_{i}=0, i=1, \ldots, m-1, Q_{1}+\cdots+Q_{m}=0\right\rangle,
$$

or simply,

$$
G \cong\left\langle Q_{1}, \ldots, Q_{m-1} \mid \alpha_{i} Q_{i}=0, i=1, \ldots, m-1\right\rangle .
$$

Since $\alpha_{i} \neq 0$ for $i=1, \ldots, m-1$, the group $G \cong \mathbf{Z}$ is finite. This contradiction tells us that $S^{2} \times S^{1}$ cannot be obtained in this manner and hence $F$ must be nonorientable.

The first homology of an orientable generalized Seifert fibered manifold with a nonorientable orbit surface is

$$
\begin{array}{r}
G=\left\langle A_{1}, \ldots, A_{|g|}, Q_{1}, \ldots, Q_{m}, H\right| \alpha_{i} Q_{i}+\beta_{i} H=0, i=1, \ldots, m \\
\left.2 H=0, Q_{1}+\cdots+Q_{m}+2 A_{1}+\cdots+2 A_{|g|}=0\right\rangle .
\end{array}
$$

Again, without loss of generality, $\alpha_{i} \neq 0$ for $i=1, \ldots, m-1$ and $\alpha_{m}=0$, $\beta_{m}=1$. Again $H=0$ and we get

$$
\begin{aligned}
G \cong\left\langle A_{1}, \ldots, A_{|g|}, Q_{1}, \ldots, Q_{m}\right| \alpha_{i} Q_{i}=0, i=1, \ldots, m-1 \\
\left.Q_{1}+\cdots+Q_{m}+2 A_{1}+\cdots+2 A_{|g|}=0\right\rangle \\
\cong\left\langle A_{1}, \ldots, A_{|g|}, Q_{1}, \ldots, Q_{m-1} \mid \alpha_{i} Q_{i}=0, i=1, \ldots, m-1\right\rangle \\
\cong \mathbf{Z}^{|g|} \oplus\left\langle Q_{1}, \ldots, Q_{m-1} \mid \alpha_{i} Q_{i}=0, i=1, \ldots, m-1\right\rangle .
\end{aligned}
$$

Since we know that $G \cong \mathbf{Z}$ and the remaining $\alpha_{i}$ 's are nonzero we must have $g=-1$ and $\alpha_{1}=\alpha_{2}=\cdots=\alpha_{m-1}=1$. In terms of this generalized Seifert manifold construction, we have

$$
S^{2} \times S^{1} \cong M\left(-1 ;\left(1, \beta_{1}\right), \ldots,\left(1, \beta_{m-1}\right),(0,1)\right) .
$$

But whenever $\alpha_{i}=1$ the corresponding surgery does not add a new exceptional fiber. Therefore,

$$
S^{2} \times S^{1} \cong M(-1 ;(0,1))
$$




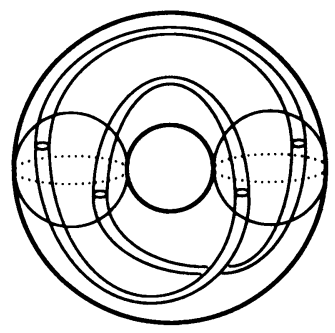

FIGURE 1. A twisted $S^{1}$ bundle over a Möbius band is homeomorphic to $S^{2} \times S^{1}$ minus a regular neighborhood of a $(2,1)$-torus knot.

Let us consider $M(-1 ;(0,1))$ for a moment. We are attaching the solid torus, $V$, to a twisted $S^{1}$-bundle over a Möbius band, so that a meridian is identified to a fiber. Another way of viewing this twisted $S^{1}$-bundle is as a twisted annulus bundle over a circle. (See Figure 1.)

As we travel around the $S^{1}$-factor the boundary components become interchanged. From this viewpoint we see that this is $S^{2} \times S^{1}$ minus a regular neighborhood of a $(2,1)$-torus knot. This regular neighborhood is $V$, and its core $K$ is therefore a $(2,1)$-torus knot. The fibers of $M$, from the fibration $M(-1 ;(0,1))$, are parallel circles on the annuli. However, we may re-fiber $S^{2} \times S^{1}$ so that $K$ is an ordinary fiber. That is,

$$
M(-1 ;(0,1)) \cong M(0 ;(2,1),(2,-1)) \text {. }
$$

Thus we have our result.

Lemma 5. If $L$ is a two-bridge link and $u(L)=1$, then for some Seifert fibering of $S^{2} \times S^{1}, M_{L}$ is obtained by $r$-surgery along an ordinary fiber, where $\Delta(r, \mu)=$ 2.

Proof. From Lemma 3 we know that $M_{L}$ is obtained by $r$-surgery on some knot $K$ in $S^{2} \times S^{1}$, where $\Delta(r, \mu)=2$. Let $M=S^{2} \times S^{1}-N(K)$.

$$
\begin{array}{cll}
M(\mu)=S^{2} \times S^{1} & \text { and } & \pi_{1}\left(S^{2} \times S^{1}\right)=\mathbf{Z} . \\
M(r)=M_{L} & \text { and } & \pi_{1}\left(M_{L}\right)=\mathbf{Z}_{q}
\end{array}
$$

since $M_{L}$ is the lens space $L(q, p)$ for $L=S(q, p)$. By the Cyclic Surgery Theorem [CGLS] this is impossible unless $M$ is Seifert fibered. Lemma 4 implies that $K$ is an ordinary fiber in some Seifert fibration.

Before stating the next lemma, we establish some additional notation. Let $T=S^{1} \times D^{2}$ with $\mu$ and $\lambda$ denoting a standard meridian and longitude, respectively, on $\partial T$. Let $C_{p, q} \subset T$ be a $(p, q)$-curve on $W=S^{1} \times \frac{1}{2} D^{2}$. For nontriviality $q \geq 2$. $T$ has a Seifert fibration in which $C_{p, q}$ is an ordinary 
fiber. Let $N$ be a 0 -framed tubular neighborhood of $C_{p, q}$ in $S^{1} \times D^{2}$. For $r$ a slope on $\partial N$, using the usual meridian-longitude coordinates, $r=s / t \in$ $\mathbf{Q} \cup\{\infty\}$, with $s$ and $t$ relatively prime.

Let $V$ be another solid torus with meridian $\mu_{V}$. Identifying $\partial V$ with $\partial T$ so that $\mu_{V}$ is attached to $\mu$ results in $S^{2} \times S^{1}$, the fibration of $T$ extends to all of $S^{2} \times S^{1}$, the Seifert invariant being $M(0 ;(p, q),(p,-q))$.

In the following lemma we will be performing $r$-surgery along an ordinary fiber of $S^{2} \times S^{1}$ as described above. Again, we will use $M(r)$ to denote this manifold.

Lemma 6.

$$
M(r) \cong \begin{cases}L(q, p) \# L(q, p) & \text { if } r=p q, \\ L\left(t q^{2}, s\right) & \text { if } s=t p q \pm 1,\end{cases}
$$

and otherwise is a Seifert fiber space with orbit surface $S^{2}$ and 3 singular fibers of multiplicities $q, q$, and $|t p q-s|$.

Proof. Let $Y=S^{2} \times S^{1}-N . Y$ is Seifert fibered with orbit surface a disk and 2 singular fibers of multiplicity $q$. Ordinary fibers on $\partial N$ are $(p q, 1)$-curves. If $r \neq p q$, the Seifert fibration extends to $M(r)$. The orbit surface is $S^{2}$. There are 2 or 3 singular fibers; the original two, both of multiplicity $q$, and possibly a third of multiplicity $|t p q-s|$. This is the intersection number of an $(s, t)$-curve with a $(p q, 1)$-curve on $\partial N$. If $|t p q-s|>1$, there are three singular fibers.

When $t p q-s= \pm 1$ there are only two singular fibers. To calculate $M(r)$ in this instance we shall begin with $T$, perform the surgery along $C_{p, q}$ and then attach $V$. We know that the surgery on $T$ results in another solid torus [G]. To calculate $M(r)$ we need only find the image of $\mu$ after the surgery. These surgeries correspond to Dehn twistings along an annulus bounded by $C_{p, q}$ and a $(p, q)$-curve on $\partial T$. A standard meridional disk is punctured $q$ times by $C_{p, q}$. At each puncture an integral twist adds $p$ copies of $\mu$ and $q$ copies of $\lambda$ to $\mu$. Thus after $\pm t$ twists $\mu$ has become $(1 \pm t p q) \mu+\left(t q^{2}\right) \lambda$. Hence $M(r)$ is homeomorphic to the lens space $L\left(t q^{2}, s\right)$, since we are assuming that $t p q-s= \pm 1$.

Finally we must calculate $M(r)$ when $r=p q$. Recall $W=S^{1} \times \frac{1}{2} D^{2} \subset T$. $C_{p, q}$ is a $(p, q)$-curve on $W . Y$ is the union of the solid tori $W \cup_{A} Z$, where $Z$ is a collared $V$, and $A$ is an annular neighborhood of a $(p, q)$-curve on both $\partial W$ and $\partial Z$. Also, $\partial A$ is a pair of ordinary fibers, $(p q, 1)$-curves on $\partial N$. These curves separate $\partial N$ into two annuli $A_{W}$ and $A_{Z}$ where $A_{W} \subset \partial W$ and $A_{Z} \subset \partial Z . \quad M(r) \cong\left(W \cup_{A} Z\right) \cup V$. Since $r=p q, \partial N$ and $\partial V$ are being identified in such a way that every fiber on $\partial N$ bounds a disk in $V$. In particular, $\partial A$ bounds two disjoint disks $D_{1}$ and $D_{2}$ in $V$. These disks divide $V$ into two 3-balls $B_{W}$ and $B_{Z}$, chosen so that the 2-sphere $A \cup D_{1} \cup D_{2}$ separates $M(r)$ into $W \cup B_{W}$ and $Z \cup B_{Z} . B_{W}$ is attached as a 2-handle to $W$ along $A_{W}$. Thus $W \cup B_{W}$ is a punctured $L(q, p)$. Similarly, $Z \cup B_{Z}$ is also a punctured $L(q, p)$. Therefore, $M(r) \cong L(q, p) \# L(q, p)$. 


\section{SOME WORK WITH CONTINUED FRACTIONS AND THE EULER BRACKET FUNCTION}

(See [Rob] for a fuller exposition.)

Recall by $\left[a_{0}, a_{1}, \ldots, a_{r}\right]$ we mean the continued fraction

$$
a_{0}+\frac{1}{a_{1}+\frac{1}{a_{2}+\frac{1}{a_{3}+\cdot \cdot+\frac{1}{a_{r}}}}}
$$

The Euler bracket function $E\left[x_{0}, \ldots, x_{k}\right]$ is the sum of all products obtained from the product $1 \cdot x_{0} x_{1} \cdots x_{k}$ by omitting zero or more disjoint pairs of consecutive factors $x_{i} x_{i+1}$. For example $E\left[x_{0}, x_{1}, x_{2}\right]=x_{0} x_{1} x_{2}+x_{0}+x_{2}$.

By convention, we define $E[]=E[\varnothing]=1$ and $E\left[x_{j}, \ldots, x_{k}\right]=1$ if $k<j$. The following facts about the Euler bracket function can easily be shown and are used in the proof of the following lemma.

(1) $E\left[x_{0}, \ldots, x_{n}\right]=x_{n} E\left[x_{0}, \ldots, x_{n-1}\right]+E\left[x_{0}, \ldots, x_{n-2}\right]$, for $n>0$;

$$
\begin{gathered}
E\left[x_{0}, \ldots, x_{n}\right]=E\left[x_{n}, \ldots, x_{0}\right] ; \\
E\left[-x_{0}, \ldots,-x_{n}\right]=(-1)^{n+1} E\left[x_{0}, \ldots, x_{n}\right] ; \\
{\left[x_{0}, \ldots, x_{n}\right]=\frac{E\left[x_{0}, \ldots, x_{n}\right]}{E\left[x_{1}, \ldots, x_{n}\right]} .}
\end{gathered}
$$

In addition, $E\left[x_{0}, x_{1}, \ldots, x_{n}\right]$ and $E\left[x_{1}, \ldots, x_{n}\right]$ are coprime.

\section{Lemma 7.}

(i) $E\left[a_{0}, \ldots, a_{k}, x,-a_{k}, \ldots,-a_{0}\right]=(-1)^{k+1} x E\left[a_{0}, \ldots, a_{k}\right]^{2}$.

(ii) $E\left[a_{1}, \ldots, a_{k}, x,-a_{k}, \ldots,-a_{0}\right]$

$$
=(-1)^{k+1} x E\left[a_{1}, \ldots, a_{k}\right] E\left[a_{0}, \ldots, a_{k}\right]+1 \text {. }
$$

As an immediate corollary, we have

\section{Corollary 8.}

$$
\left[a_{0}, \ldots, a_{k}, x,-a_{k}, \ldots,-a_{0}\right]=\frac{(-1)^{k+1} x E\left[a_{0}, \ldots, a_{k}\right]^{2}}{(-1)^{k+1} x E\left[a_{1}, \ldots, a_{k}\right] E\left[a_{0}, \ldots, a_{k}\right]+1} .
$$

Proof of Lemma 7. The proof is by induction on $k$.

(i) is true for $k=0$ :

$$
E\left[a_{0}, x,-a_{0}\right]=-x a_{0}^{2}=(-1)^{1} x E\left[a_{0}\right]^{2} .
$$

(ii) is true for $k=0$ :

$$
E\left[x,-a_{0}\right]=-x a_{0}+1=(-1)^{1} x E[] E\left[a_{0}\right]+1 .
$$


Now, assume both statements are true for $k=n-1$; that is,

$$
\begin{gathered}
E\left[a_{0}, \ldots, a_{n-1}, x,-a_{n-1}, \ldots,-a_{0}\right]=(-1)^{n} x E\left[a_{0}, \ldots, a_{n-1}\right]^{2} ; \\
E\left[a_{1}, \ldots, a_{n-1}, x,-a_{n-1}, \ldots,-a_{0}\right] \\
=(-1)^{n} x E\left[a_{1}, \ldots, a_{n-1}\right] E\left[a_{0}, \ldots, a_{n-1}\right]+1 .
\end{gathered}
$$

For $k=n$ :

$$
\begin{aligned}
& E\left[a_{0}, \ldots, a_{n}, x,-a_{n}, \ldots,-a_{0}\right] \\
&=-a_{0} E\left[a_{0}, \ldots, a_{n}, x,-a_{n}, \ldots,-a_{1}\right]+E\left[a_{0}, \ldots, a_{n}, x,-a_{n}, \ldots,-a_{2}\right] \\
&=-a_{0}\left(a_{0} E\left[a_{1}, \ldots, a_{n}, x,-a_{n}, \ldots,-a_{1}\right]+E\left[a_{2}, \ldots, a_{n}, x,-a_{n}, \ldots,-a_{1}\right]\right) \\
&+a_{0} E\left[a_{1}, \ldots, a_{n}, x,-a_{n}, \ldots,-a_{2}\right]+E\left[a_{2}, \ldots, a_{n}, x,-a_{n}, \ldots,-a_{2}\right] \\
&=-a_{0}^{2} E\left[a_{1}, \ldots, a_{n}, x,-a_{n}, \ldots,-a_{1}\right]-a_{0} E\left[a_{2}, \ldots, a_{n}, x,-a_{n}, \ldots,-a_{1}\right] \\
&+a_{0} E\left[a_{1}, \ldots, a_{n}, x,-a_{n}, \ldots,-a_{2}\right]+E\left[a_{2}, \ldots, a_{n}, x,-a_{n}, \ldots,-a_{2}\right] \\
&=-a_{0}^{2}(-1)^{n} x E\left[a_{1}, \ldots, a_{n}\right]^{2}-a_{0}\left((-1)^{n} x E\left[a_{2}, \ldots, a_{n}\right] E\left[a_{1}, \ldots, a_{n}\right]+1\right) \\
&+a_{0} E\left[-a_{2}, \ldots,-a_{n}, x, a_{n}, \ldots, a_{1}\right]+(-1)^{n+1} x E\left[a_{2}, \ldots, a_{n}\right]^{2} \\
&= a_{0}^{2}(-1)^{n+1} x E\left[a_{1}, \ldots, a_{n}\right]^{2}+a_{0}(-1)^{n+1} x E\left[a_{2}, \ldots, a_{n}\right] E\left[a_{1}, \ldots, a_{n}\right]-a_{0} \\
&+a_{0}\left((-1)^{n} x E\left[-a_{2}, \ldots,-a_{n}\right] E\left[-a_{1}, \ldots,-a_{n}\right]+1\right)+(-1)^{n+1} x E\left[a_{2}, \ldots, a_{n}\right]^{2} \\
&= a_{0}^{2}(-1)^{n+1} x E\left[a_{1}, \ldots, a_{n}\right]^{2}+a_{0}(-1)^{n+1} x E\left[a_{2}, \ldots, a_{n}\right] E\left[a_{1}, \ldots, a_{n}\right]-a_{0} \\
&+a_{0}(-1)^{n+1} x E\left[a_{2}, \ldots, a_{n}\right] E\left[a_{1}, \ldots, a_{n}\right]+a_{0}+(-1)^{n+1} x E\left[a_{2}, \ldots, a_{n}\right]^{2} \\
&=(-1)^{n+1} x\left(a_{0}^{2} E\left[a_{1}, \ldots, a_{n}\right]^{2}+2 a_{0} E\left[a_{2}, \ldots, a_{n}\right] E\left[a_{1}, \ldots, a_{n}\right]+E\left[a_{2}, \ldots, a_{n}\right]^{2}\right) \\
&=(-1)^{n+1} x\left(a_{0} E\left[a_{1}, \ldots, a_{n}\right]+E\left[a_{2}, \ldots, a_{n}\right]\right)^{2} \\
&=(-1)^{n+1} x E\left[a_{0}, \ldots, a_{n}\right]^{2} .
\end{aligned}
$$

This establishes (i).

For (ii):

$$
\begin{aligned}
& E\left[a_{1}, \ldots, a_{n}, x,-a_{n}, \ldots,-a_{0}\right] \\
& =-a_{0} E\left[a_{1}, \ldots, a_{n}, x,-a_{n}, \ldots,-a_{1}\right]+E\left[a_{1}, \ldots, a_{n}, x,-a_{n}, \ldots,-a_{2}\right] \\
& =-a_{0}(-1)^{n} x E\left[a_{1}, \ldots, a_{n}\right]^{2}+(-1)^{n} x E\left[-a_{2}, \ldots,-a_{n}\right] E\left[-a_{1}, \ldots,-a_{n}\right]+1 \\
& =a_{0}(-1)^{n+1} x E\left[a_{1}, \ldots, a_{n}\right]^{2}+(-1)^{n+1} x E\left[a_{2}, \ldots, a_{n}\right] E\left[a_{1}, \ldots, a_{n}\right]+1 \\
& =(-1)^{n+1} x E\left[a_{1}, \ldots, a_{n}\right]\left(a_{0} E\left[a_{1}, \ldots, a_{n}\right]+E\left[a_{2}, \ldots, a_{n}\right]\right)+1 \\
& =(-1)^{n+1} x E\left[a_{1}, \ldots, a_{n}\right] E\left[a_{0}, \ldots, a_{n}\right]+1 .
\end{aligned}
$$

The following will also be used in the proof of Theorem 2.

\section{Lemma 9.}

(i) $E\left[b_{0}, b_{1}, \ldots, b_{m-1}, b_{m}, x,-a_{k},-a_{k-1}, \ldots,-a_{1},-a_{0}\right]$

$$
=(-1)^{k+1} E\left[b_{0}, b_{1}, \ldots, b_{m-1}, b_{m}, x-1,1, a_{k}-1, a_{k-1}, \ldots, a_{1}, a_{0}\right] \text {. }
$$

(ii) $E\left[b_{0}, b_{1}, \ldots, b_{m-1}, b_{m},-x,-a_{k},-a_{k-1}, \ldots,-a_{0}\right]$

$$
=(-1)^{k} E\left[b_{0}, b_{1}, \ldots, b_{m-1}, b_{m}-1,1, x-1, a_{k}, a_{k-1}, \ldots, a_{1}, a_{0}\right] \text {. }
$$


The following corollary is an immediate consequence of Lemma 9.

Corollary 10. For $a_{k} \geq 2$,

$$
\begin{gathered}
{\left[a_{0}, a_{1}, \ldots, a_{k}, 2,-a_{k},-a_{k-1}, \ldots,-a_{0}\right]} \\
\quad=\left[a_{0}, a_{1}, \ldots, a_{k}, 1,1, a_{k}-1, a_{k-1}, \ldots, a_{0}\right] . \\
{\left[a_{0}, a_{1}, \ldots, a_{k-1}, a_{k},-2,-a_{k},-a_{k-1}, \ldots,-a_{0}\right]} \\
\quad=\left[a_{0}, a_{1}, \ldots, a_{k-1}, a_{k}-1,1,1, a_{k}, a_{k-1}, \ldots, a_{0}\right] .
\end{gathered}
$$

Proof of Lemma 9. Using equations (1), (2) and (3), it may be demonstrated that

$$
E\left[-a_{k},-a_{k-1}, \ldots,-a_{0}\right]=(-1)^{k+1} E\left[1, a_{k}-1, a_{k-1}, \ldots, a_{0}\right]
$$

and

(6) $E\left[x,-a_{k},-a_{k-1}, \ldots,-a_{0}\right]=(-1)^{k+1} E\left[x-1,1, a_{k}-1, a_{k-1}, \ldots, a_{0}\right]$

(i) This follows from equations (1), (5) and (6), and by using induction on $m$.

(ii) This may be proved using part (i).

\section{THE PROOF OF THEOREM 1}

(i) implies (ii). Let $L=S(a, b)$ with $u(L)=1$. By Lemma 5 there is an ordinary fiber $K$ of $S^{2} \times S^{1}$ such that $r$-surgery along $K$ results in the lens space $L(a, b)$, where $\Delta(r, \mu)=2$. Let $K$ be an $(m, n)$-fiber. Recall that if $N$ is a regular neighborhood of $K$, ordinary fibers on $\partial N$ are $(m n, 1)$-curves. We have that $\Delta(m n, r)=|2 m n-p|$, where $r=p / 2$ in the usual meridianlongitude coordinates on $\partial N(K)$. But $|2 m n-p|=1$, since our resulting manifold is a lens space. (Otherwise we would have three singular fibers. Lens spaces can be fibered with at most two.) By Lemma $6, L(a, b)$ is equivalent to $L\left(2 n^{2}, p\right)$, but $|2 m n-p|=1$ implies that $p=2 m n \pm 1$. Therefore, $L(a, b)$ is equivalent to $L\left(2 n^{2}, 2 m n \pm 1\right)$, where $(m, n)=1$. So $L$ is equivalent to $S\left(2 n^{2}, 2 m n \pm 1\right)$.

(ii) implies (iii). For every $\frac{n}{m} \in \mathbf{Q}$ there is a continued fraction such that $\frac{n}{m}=\left[a_{0}, \ldots, a_{k}\right]$. From Corollary 8

$$
\left[a_{0}, a_{1}, \ldots, a_{k}, \pm 2,-a_{k}, \ldots,-a_{1},-a_{0}\right]=\frac{ \pm 2(-1)^{k+1} n^{2}}{ \pm 2(-1)^{k+1} m n+1} .
$$

Choosing the appropriate sign $( \pm 2)$ gives the required result. Therefore $L$ is equivalent to $C\left(a_{0}, a_{1}, \ldots, a_{k}, \pm 2,-a_{k}, \ldots,-a_{1},-a_{0}\right)$. 


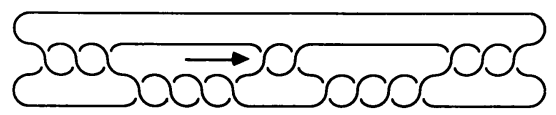

Figure 2. $C(3,-4,-2,4,-3)=S(242,87)$

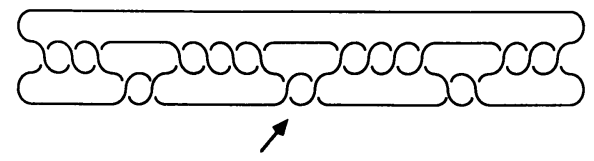

Figure 3. $C(3,2,4,2,-4,-2,-3)=S(1922,557)$

(iii) implies (i). The symmetry of any such two-bridge link shows that $u(L)=$ 1. See Figure 2 for a case where $k$ is odd. Changing one of the crossings at the arrow results in a trivial link with two components.

For $k$ even we have a similar situation. See Figure 3. If we change either of the crossings at the arrow, again we will obtain a trivial link.

(iii) implies (iv). We are assuming that $L$ may be expressed as

$$
C\left(a_{0}, a_{1}, \ldots, a_{k}, \pm 2,-a_{k}, \ldots,-a_{1},-a_{0}\right) \text {. }
$$

By Corollary 8 ,

$$
\left[a_{0}, a_{1}, \ldots, a_{k}, \pm 2,-a_{k}, \ldots,-a_{1},-a_{0}\right]=\frac{ \pm 2 v^{2}}{ \pm 2 v w+1},
$$

where $v$ and $w$ are relatively prime. If $v=0, L$ is the trivial link of two components. Therefore we may assume $v \geq 1$.

There are three cases we need to consider:

(1) $v=1 . L$ is equivalent to a Hopf link, $C(2)=C(1,1)$.

(2) $v>|w| \geq 1$. Using the Euclidean algorithm $\left|\frac{v}{w}\right|=\left[c_{0}, c_{1}, \ldots, c_{r}\right]$ with $c_{i} \geq 1$ for $i=0,1, \ldots, r$ and $c_{r} \geq 2$.

If $\frac{v}{w}>0$, by Corollary 8 and [S],

$$
L=C\left(c_{0}, c_{1}, \ldots, c_{r}, \pm 2,-c_{r}, \ldots,-c_{1},-c_{0}\right),
$$

where the sign on the \pm 2 here may differ from that in (7). By Corollary 10,

$$
\begin{aligned}
& L=C\left(c_{0}, c_{1}, \ldots, c_{r-1}, c_{r}, 1,1, c_{r}-1, c_{r-1}, \ldots, c_{1}, c_{0}\right) \text { or } \\
& L=C\left(c_{0}, c_{1}, \ldots, c_{r-1}, c_{r}-1,1,1, c_{r}, c_{r-1}, \ldots, c_{1}, c_{0}\right) .
\end{aligned}
$$

If $\frac{v}{w}<0$, then $\frac{v}{w}=\left[-c_{0},-c_{1}, \ldots,-c_{r}\right]$. As above,

$$
L=C\left(-c_{0},-c_{1}, \ldots,-c_{r}, \pm 2, c_{r}, \ldots, c_{1}, c_{0}\right) \text {. }
$$

But, it is clear from the diagrams that for $n$ even, $C\left(x_{0}, x_{1}, \ldots, x_{n}\right)$ and $C\left(x_{n}, \ldots, x_{1}, x_{0}\right)$ are equivalent. Thus

$$
L=C\left(c_{0}, c_{1}, \ldots, c_{r}, \pm 2,-c_{r}, \ldots,-c_{1},-c_{0}\right) .
$$

We finish as above. 


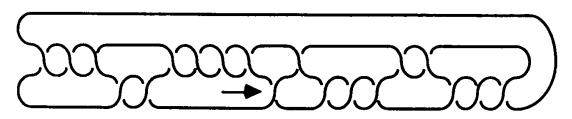

Figure 4. $C(3,2,4,1,1,3,2,3)=S(1922,557)$

(3) $|w|>v>1$. By the division algorithm there are integers $d$ and $z$ such that $w=d v+z$ and $0<z<v$. Note that $z \geq 1$, since $v>1$ and $v$ and $w$ are coprime.

$$
\pm 2 v w+1= \pm 2 v(d v+z)+1 \equiv \pm 2 v z+1 \quad\left(\bmod 2 v^{2}\right) .
$$

Therefore by [S],

$$
L=S\left( \pm 2 v^{2}, \pm 2 v w+1\right)=S\left( \pm 2 v^{2}, \pm 2 v z+1\right) .
$$

We find ourselves in case 2.

(iv) implies (iii). This is an immediate consequence of Corollary 10. As an example of the equivalence of conditions (iii) and (iv), see Figure 4. The link in Figure 4 is isotopic to the link in Figure 3.

Remark. The proof that conditions (iii) and (iv) of Theorem 2 are equivalent is analogous to those implications above.

\section{ADDENDUM}

We may use Theorems 1 and 2 to prove the following corollary:

Corollary 11. Let $K$ be a two-bridge knot or link with $u(K)=1$. There is a crossing in a minimal diagram of $K$ which, when changed, unknots $K$.

Proof. We express $K$ as in condition (iv) of Theorem 1 or 2 . Since each entry in these expansions has the same sign, this is an alternating diagram. By [Mu] these are minimal diagrams. We examine the knot

$$
C\left(c_{0}, c_{1}, \ldots, c_{r}, 1^{*}, 1, c_{r}-1, c_{r-1}, \ldots, c_{1}\right),
$$

The other cases are similar. If we change the crossing in the starred position we obtain the knot

$$
K^{\prime}=C\left(c_{0}, c_{1}, \ldots, c_{r},-1,1, c_{r}-1, c_{r-1}, \ldots, c_{1}\right) .
$$

But we may compute that the continued fraction,

$$
\left[c_{0}, c_{1}, \ldots, c_{r},-1,1, c_{r}-1, c_{r-1}, \ldots, c_{1}\right]=\frac{ \pm 1}{0} .
$$

Thus $K^{\prime}$ is unknotted.

Corollary 11 leads us to propose the following: 
Conjecture 12. Let $K$ be a knot or link with $u(K)=1$. There is a crossing in a minimal diagram of $K$ which, when changed, unknots $K$.

Using $[\mathrm{N}]$ for knots and his own calculations for links, the author has verified this conjecture for knots and links with fewer than ten crossings.

We now examine the linking number of two-bridge links with unlinking number one. Let $\theta$ and $\phi$ be the components of a two-component link, $L$. Let $\operatorname{lk}(\theta, \phi)$ be the absolute value of the linking number of $\theta$ and $\phi$. If $u(L)=1$, it is clear that $1 \mathrm{k}(\theta, \phi)=0$ or 1 , since a single crossing change can reduce $\operatorname{lk}(\theta, \phi)$ by at most one. For example in the Whitehead link, $C(2,1,1,1)=C(2,2,-2)=S(8,3), \operatorname{lk}(\theta, \phi)=0$. For the Hopf link, $C(2)=S(2,1), \operatorname{lk}(\theta, \phi)=1$.

For unlinking number one two-bridge links, we have the following corollary to Theorem 1.

Corollary 13. If $L=S\left(2 n^{2}, 2 m n \pm 1\right)$, then

$$
\operatorname{lk}(\theta, \phi)= \begin{cases}0 & \text { if } n \text { is even, } \\ 1 & \text { if } n \text { is odd. }\end{cases}
$$

Proof. The proof of Theorem 1 implies that

$$
L=C\left(c_{0}, c_{1}, \ldots, c_{r}, \pm 2,-c_{r}, \ldots,-c_{1},-c_{0}\right)
$$

for some positive integers $c_{0}, c_{1}, \ldots, c_{r}$. In addition $c_{r} \geq 2$. Without loss of generality, we may assume that $r$ is even, since

$$
\begin{aligned}
& C\left(c_{0}, c_{1}, \ldots, c_{r}, \pm 2,-c_{r}, \ldots,-c_{1},-c_{0}\right) \\
& \quad=C\left(c_{0}, c_{1}, \ldots, c_{r}-1,1, \mp 2,-1,-c_{r}+1, \ldots,-c_{1},-c_{0}\right) .
\end{aligned}
$$

From the symmetry of the diagram for $L$, we see that the only possible contribution to $1 \mathrm{k}(\theta, \phi)$ occurs at the "central \pm 2 half-twists."

When $n$ is even, $S(n, m)=C\left(a_{0}, a_{1}, \ldots, a_{k}\right)$ is a two-component link. For $n$ odd, $S(n, m)$ is a knot. An examination of cases using the above facts shows that the central crossings involve exactly one of the components when $n$ is even, giving $\mathrm{lk}(\theta, \phi)=0$. Both components are involved when $n$ is odd, so $\operatorname{lk}(\theta, \phi)=1$.

Remark. For any two-bridge link there is an involution of $S^{3}$ exchanging the components of the link. Thus for $n$ even in the above corollary, the crossing change can occur in either of the components.

\section{REFERENCES}

[BZ] G. Burde and H. Zieschang, Knots, de Gruyter Studies in Mathematics, no. 5, Walter de Gruyter, Berlin, 1985.

[C] J. H. Conway, An enumeration of knots and links and some of their related properties, Computational Problems in Abstract Algebra (Proc. Conf. Oxford) (J. Leech, ed.), Pergamon Press, New York, 1969, pp. 329-358. 
[CGLS] M. Culler, C.McA. Gordon, J. Luecke, and P. Shalen, Dehn surgery on knots, Ann. of Math. (2) 125 (1987), 237-300.

[G] C. McA. Gordon, Dehn surgery and satellite knots, Trans. Amer. Math. Soc. 275 (1983), 687-708.

[JN] M. Jankins and W. D. Neumann, Lectures on Seifert manifolds, Brandeis Lecture Notes, 1983.

[KM] T. Kanenobu and H. Murakami, Two-bridge knots with unknotting number one, Proc. Amer. Math. Soc. 98 (1986), 499-502.

[L] W. B. R. Lickorish, The unknotting number of a classical knot, Combinatorial Methods in Topology and Algebraic Geometry, Contemp. Math., vol. 44 (J. R. Harper and R. Mandelbaum, eds.), Amer. Math. Soc., Providence, RI, 1985, pp. 117-121.

[M] L. Moser, Elementary surgery along a torus knot, Pacific J. Math. 38 (1971), 737-745.

[Mu] K. Murasugi, Jones polynomials and classical conjectures in knot theory, Topology 26 (1987), 187-194.

[N] Y. Nakanishi, A note on unknotting number, Math. Sem. Notes, Kobe Univ. 9 (1981), 99-108.

[Rob] J. Roberts, Elementary number theory, a problem oriented approach, MIT Press, Cambridge, MA, 1977.

[Rol] D. Rolfsen, Knots and links, Mathematics Lecture Series, no. 7, Publish or Perish, Berkeley, CA, 1976.

[S] H. Schubert, Knoten mit zwei Brücken, Math Z. 65 (1956), 133-170.

[Seif] H. Seifert, Topologie dreidimensionaler gefaserter Räume, Acta Math. 60 (1933), 147-238.

[Sieb] L. Siebenmann, Exercices sur les næu ds rationnels, preprint, Orsay, 1975.

Department of Mathematics, University of Texas, Austin, TeXas 78712 